\title{
XVIII. On the passage of the galvanic current through iron
}

\section{Felix Auerbach Ph.D.}

To cite this article: Felix Auerbach Ph.D. (1879) XVIII. On the passage of the galvanic current through iron, Philosophical Magazine Series 5, 8:47, 138-152, DOI: 10.1080/14786447908639665

To link to this article: http://dx.doi.org/10.1080/14786447908639665

曲 Published online: 13 May 2009.

Submit your article to this journal $[\pi$

Џ Article views: 2

Q View related articles $\asymp$ 
sulphonic compound, having, as a rule, the formula

$$
\mathrm{C}_{20} \mathrm{H}_{16}\left(\mathrm{C}_{6} \mathrm{H}_{5}\right)_{2} \cdot \mathrm{C}_{6} \mathrm{H}_{4} \mathrm{~N}_{3}\left[\mathrm{NaSO}_{3}\right] \text {. }
$$

Our own sample, which was prepared many years ago, may possibly have contained a little of the disulphonic compound. The solution which I employed contained 1 grm. in a litre, being therefore three hundred times as strong as in I, and II. When this was boiled, even for several hours, it refused to bleach; but on allowing the hot liquid to cool gradually, the colour slowly faded away before the ordinary temperature was reached. Repeated attempts to decolorize the liquid by heating to temperatures short of $100^{\circ}$ were all attended with failure.

When the decolorized liquid was kept for three or four hours, or frozen, or mixed with a little hydric acetate, the colour was restored.

The dissociation-temperature for Nicholson's blue is obviously a little below the boiling-point of water.

Phenomena of bleaching in connexion with Nicholson's blue were first noticed by my friend Mr. Louis Campbell.

XVIII. On the Passage of the Galvanic Current through Iron. By Felix Auerbach, Ph.D., of Breslau.

[Continued from p. 18.]

$\S 7$. T $\mathrm{N}$ the following I will endeavour to elucidate the ob1 served phenomena on the basis of the theory of rotable molecular magnets. In so doing I make use of the conception of work, defining it for the present case as the product obtained when the force which must be overcome for the rotation of a molecular magnet is multiplied by the angular quantity of that rotation. The extra currents arising in iron have already been commonly recognized as the expression of such performance of work. When, from a fixed moment onwards, a current generated by a constant electromotive force performs work which till then it did not perform, then Ohm's law is valid only on the hypothesis that either the current-intensity $i$ or the resistance $w$ obtains another value. Assuming the former case, the equation is usually written

$$
i=\frac{e-\frac{1}{a} \frac{\partial V}{\partial t}}{w} \text {; }
$$

that is, the quantity $\frac{1}{a}-\frac{\mathrm{V}}{\partial t}$ (in which $a$ denotes the value of the

* Helmholtz, Die Erhaltang der Kraft: Berlin, 1847. 
work of the unit of heat, $V$ the potential, for instance, of the unit current on the magnet in reference to which work is performed) is 'regarded' as a new electromotive force which counteracts the first. But I do not see why it is not also admissible to write

$$
i=\frac{e}{w+w_{\mathrm{A}}},
$$

where $w_{\mathrm{A}}$ denotes a new resistance added to $w$ in consequence of the external work*. At all events it cannot be proved, as Colley $\dagger$ will have it, that the latter equation is false. Colley thinks himself authorized to conclude as follows:--If T and " $\mathrm{T}^{\prime}$ denote the times necessary, with and without the performance of work, to dissolve 1 gram of zinc in the galvanic series, then, if $w$ varies,

$$
\frac{e^{2}}{w} \mathrm{~T}=\frac{e^{2}}{v+w_{\mathbf{A}}} \mathrm{T}^{\prime}+q \mathrm{~T}^{\prime}, \ldots . . .
$$

where $g$ signifies the work done in the unit of time. Now, however,

consequently

$$
\frac{e}{w} \mathrm{~T}=\frac{e}{w+w_{\mathrm{A}}} \mathrm{T}^{/} ; . . . \quad . \quad .
$$

$$
e=e+\frac{q}{e}\left(w+w_{\mathrm{A}}\right)
$$

which is impossible.

Equation (1) is true; but equation (2) is as little applicable as in the other case (where $e$ is considered variable) the corresponding equation

$$
\frac{e}{w} \mathrm{~T}=\frac{e-\epsilon}{w} \mathrm{~T}^{\prime}, \quad . \quad . \quad . \quad . \quad . \quad .
$$

where $\epsilon$ signifies the variation of $e$. Much rather are both equations to be replaced by the common equation

$$
\mathrm{T}=\mathrm{T}^{\prime}, \text {. . . . . . . . . (3) }
$$

which expresses that with $i$ the evolution of heat has also diminished; and this leads, in our case, to the very possible equation

from which we get

$$
\frac{e^{2}}{w}=\frac{e^{2}}{w+v_{\mathrm{A}}}+q
$$

$$
w_{\mathrm{A}}=\frac{w^{2} q}{e^{2}-w q} \cdot u^{2} \cdot \text {. . . . . }
$$

* But compare Wiedemarn, Galv, ii. 2, p. 521.

† Pogg. Ann. clvii. p. 370 (1876). 
and

$$
q=\frac{e^{2} w_{\mathrm{A}}}{w\left(w+w_{\mathrm{A}}\right)} \cdot \quad \cdot \quad \cdot \quad \cdot
$$

This equation can be readily verified if we pursue the extra current which arises at the closing of a known current, if the current deflects a magnetic needle of known moment, and if, besides, the horizontal component of the intensity of the earth's magnetism is known*.

In regard to practice it is at all events most convenient, whenever work is performed, whether momentary or lasting, to admit an alteration of the resistance.

Accordingly the resistance of an iron wire in the first moment after the closing must be greater, in the first moment after the opening it must be less, than during the rest of the time that the current lasts. For then the molecular magnets, in consequence of the directing force of the current, shift into a position more or less approximating to the circular arrangement when the current has to perform work in relation to the direction-force of the molecules. Here the molecules return more or less into their natural position; the direction-force therefore does work in regard to the current. With this the observations are in complete accordance.

As soon as that actual energy which the molecular magnets receive from the rotating force of the current is entirely converted into potential, the current has no more work to perform with respect to the direction of the molecular magnets. Hence we could not but conclude that the resistance would now take its true value, corresponding to the iron wire at rest internally (or in a determined thermal motion), if we had not to bear in mind that through the action of the rotating forces exerted by the current the internal state of the iron (as may also be imagined) has become different, and remains so till the current is interrupted. Accordingly the iron might possess two different resistances, of which one only, viz. that of the circularly mag-

* That even in the case represented by M. Colley ( $e$ variable) equation $(2 \mathrm{~A})$ is not applicable may be inferred from its leading to a contradiction. For $\mathrm{M}$. Colley arrives by correct conclusions at the equation (corresponding to eq. $4 \mathrm{~B}$ )

$$
\bar{q}=\frac{\epsilon(e-\epsilon)}{w}
$$

which cannot be true, since for $\epsilon$ indefinitely small it yields

$$
q=\frac{e d e}{w} \text { instead of } q=\frac{2 e d e}{w} ;
$$

while if in this case also, as above, we make use of eq. (3), it brings us to the last-mentioned, the true equation. 
netized iron, would be at once accessible to observation, while the other, resistance of the unmagnetic, would have an important theoretic signification, inasmuch as it alone is comparable with the quantities which in other metals we bluntly call the resistance.

Assuming, then, that these two quantities indeed differ (and experience shows that this is the case), yet no way based on special conclusions can be imagined in which we could decide which is the greater. In such cases considerations having for their starting-point the principle of the conservation of force have recently been frequently applied with success. Taking the same course, I place first of all a general principle which results therefrom, and which hitherto, so far as is known to me, has been expressed only for special cases. It is :-

No force can of itself bring in conditions more favourable for its own action than those which it meets with.

This needs no explanation; even the expression "of itself" has become universally familiar since it was introduced by Clausius*. The proposition in which he first made use of it, "Heat can never of itself pass over from a colder into a hotter body," is itself a special case of the above principle. Another is Lenz's law which determines the direction of the induced current. Further, here belong the facts that in solids the cubic coefficient of compression diminishes as the pressure increases, that the specific heat and the galvanic resistance of solid bodies increase with the temperature, \&c.

For the present case we may conclude from the above principle that the resistance of iron as observed by us when a current is conducted through it is greater than that ideal value. Thence, in the next place, it follows that circularly magnetized possesses a greater resistance than unmagnetic iron, at least if the amount of the circular magnetization does not exceed that which the current itself could produce. Evidently, however, we may drop this limitation; for if the circular magnetization be greater than the current itself can generate, yet there is always another intensity of current possessing this property. For this latter, therefore, the above proposition holds good. But the resistance of an iron wire possessing a definite magnetic state $\dagger$ is independent of the intensity of the current; consequently that principle is valid for any amount of circular magnetization.

It follows, further, that the resistance must be lessened by feeble longitudinal magnetizings. For, according to the above, we may assume that circularly magnetic iron conducts the

* Die mechanische Wärmtheorie, i. p. 81.

$\uparrow$ That this addition is necessary, will be shown in $\$ 8$. 
current the worse the more intense the circular magnetization is ; but by the added longitudinal magnetization a portion of the circular is annulled. In fact my experiments show, in iron as well as in steel, a diminution of the resistance in consequence of feeble magnetizings.

The behaviour is different when the longitudinally magnetizing force is great; a longitudinal magnetizing will then result from its cooperation with the circular magnetizing force of the principal current. And here the theory leaves a blank. The resistance of longitudinally magnetized iron can be less or greater than that of the unmagnetic. In the former case the resistance-function has nowhere a minimum or a maximum ; but rather the resistance constantly rises from the state of saturated longitudinal magnetism to the state of saturated circular magnetism : this case is realized, as the experiments show, in hard steel. In the other case the resistance-function has a minimum value for the unmagnetic state; my experiments have in fact yielded this result in iron and soft steel: here, to one and the same ordinate of the resistance-curve two abscissa correspond; that is, two magnetic states (namely, longitudinally and circularly magnetic) correspond to the same resistance. The conjectural form of the resistance-curves is represented in PI. I. fig. 2. This gives the explanatison of experiments 7,21 , and 25 , both so far as their result were positive, and also so far as they were negative. By making use of the laws laid down respecting magnetization by a circular current or by a spiral*, and respecting circular magnetization by the current flowing through the wire itself $\uparrow$, we can calculate what must be the ratio of the electromotive forces of the magnetizing and of the principal current (that is, the ratio $\mathrm{M}: \mathrm{H}$ ) at given values of $n, w_{0}, l$, and $d$, in order that $\delta$ (for iron and soft steel) may vanish. But as the smallness of the values of $\delta$ hardly permits this calculation to be tested, I forbear to carry it out. I will only mention that, according to the experiments, that ratio appears to depend not only on $n, v_{0}, l$, and $d$, but also on the nature of the wire. 'That the dependence on the thickness is considerable can be made evident by the following consideration. The longitudinally magnetic moment taken by the wire is, with equal magnetizing forces, nearly proportional to the square root of the thickness $\neq$. The case is different with the circularly magnetic moment; indeed, with equal magnetizing force the circularly magnetic moment appears not to depend essentially on the thickness: but the

* Conf. Wiedemann, Galw. ii. 1, pp. 180,320.

$\dagger$ Streintz, l. c.

† Dub, Electromagnetismus (1861), p. 197. 
magnetizing force varies considerably with the thickness ; for it is

$$
\mathrm{P}=c \int_{0}^{2 \pi} \int_{0}^{l} \int_{0}^{\frac{d}{2}} \frac{r^{2}}{d^{2}} i d r d l d \phi .
$$

Integrating, and using the equation

$$
i=\frac{\mathrm{H}}{w_{k}+\frac{4 f}{\pi d^{2}} l},
$$

in which $f$ signifies the specific resistance of iron, we get for the magnetic moment :-

$$
\mathrm{K}=\frac{\text { const. } \mathrm{H} l d}{w_{k}+\frac{4 f}{\pi d^{2}} l} \cdot \phi(d) \cdot \psi(l),
$$

where $\phi(d)$ is a complete function of $d$, slowly varying as $d$ varies, and in the same direction, and $\psi(l)$ is a function of $l$ which is of no consequence here. For larger values of $l$, at least if that of $d$ is not too great, we can so arrange that $w_{k}$ may be neglected; we then find :-

$$
\mathrm{K}=\text { const. } \mathrm{H} d^{3} \phi(d) \cdot \Psi(l) .
$$

But even when $d$ is so great that conversely $\frac{4 f l}{\pi d^{2}}$ can be neglected against $v_{k}$, still

$$
\mathrm{K}=\text { const. } \mathrm{H} d \phi(d) \cdot l \psi(l),
$$

while yet, as mentioned, the longitudinally magnetic moment is

$$
\mathrm{K}^{\prime}=\text { const. } \mathrm{M} n \sqrt{\bar{d}} \chi(l) .
$$

As the expression of the work done by the current in the rotation of the molocular magnets, an extra current occurs at the closing of the principal current, as we have seen, or, as it was expressed, a passing augmentation of the resistance. If the wire has been previously magnetized longitudinally, and is still in that state at the closing of the principal current, the rotation by the latter is of course much less. From this we might at first be inclined to conclude that the work also is less, which would be contradicted by the observed fact that the extra current is in this case more intense. But we must bear in mind that the rotation is smaller in amount because the longitudinally magnetizing force holds back the molecules more strongly than the direction-force, which alone, in the first case, counteracted the force of the principal current, but the other factor of the product representing the work, the longitudinally 
magnetizing force, is much greater than the direction-force. Now, as long as the rotations are infinitesimal, the force varies in inverse proportion to the angle of rotation; therefore the work of the current remains constant ; but when the longitudinally magnetizing force is considerable, and therefore the rotations into the axial position cannot be regarded as infinitesimal, the work to be done by the current increases, although the circular turning produced by it is less. If, to demonstrate this, we denote by $\mathrm{D}$ the direction-force, by $\mathrm{H}$ the directing force of the principal current, we get (first, apart from a longitudinal magnetizing), for the work to be done for any one molecule in rotating it the angle $\psi$ :-

$$
A=\int_{0} D \sin \psi d \psi \text {. }
$$

Now, if the direction of $\mathrm{D}$ for this molecule makes with the axis of the wire the angle $\phi$, then $\psi$ is determined by the equation

$$
D \sin \psi=M \cos (\phi+\psi)
$$

from which follows

$$
\tan \psi=\frac{\mathrm{H} \cos \phi}{\mathrm{D}+\mathrm{H} \sin \phi} .
$$

Inserting this value in the equation

we find

$$
\mathrm{A}=\mathrm{D}(1-\cos \psi) \text {, }
$$

$$
\mathrm{A}=\mathrm{D}\left(1-\frac{\mathrm{D}+\mathrm{H} \sin \phi}{\mathrm{W}}\right)
$$

where $\mathrm{W}$ denotes the quantity $+\sqrt{\mathrm{D}^{2}+2 \mathrm{HD} \sin \phi+\mathrm{H}^{2}}$.

If now we would describe rigorously the phenomena of the extra currents, we have to solve the following problems:-

(1) What is the mean value of $A$ for all the molecules of the wire?

(2) What is the amount of the corresponding work for one molecule, on which, beside the forces $\mathrm{D}$ and $\mathrm{H}$, the force $\mathrm{M}$ acts perpendicular to $H$ ?

(3) What is the mean value of this work for all the molecules?

I have prosecuted this calculation under the following assumptions:- (a) In the unmagnetic state, all the values of $\phi$ between 0 and $\frac{\pi}{2}$ are represented with equal frequency; values between $\frac{\pi}{2}$ and $\pi$ appeared to me, on account of the 
unstable equilibrium which would then of necessity prevail in certain parts of the wire, very unlikely; moreover the value of the integral, so far as it comes into consideration, is independent of these. (b) The differences of direction of the molecules will quickly diminish with $M I$ increasing, consequently with diminishing deviation from the axis of the wire ; that is, the integral which, divided by the difference of its limits, represents that mean value will be included within limits rapidly approaching one another, and at values of $M$ which are great in comparison with $\mathrm{D}$ can be replaced by a differential, and consequently that quotient by a differential quotient; the final value of it then represents the extreme value of the work of the current for saturated longitudinal magnetism.

The work is, in the second case, greater than in the first, from two causes:-first, because the work increases with the force to be overcome; an understanding of this can be obtained, without a knowledge of those general expressions, in the following manner: we have

$$
\frac{\partial \mathrm{A}}{\partial \overline{\mathrm{D}}}=1-\frac{2 \mathrm{D}+\mathrm{H} \sin \phi}{\mathrm{W}}+\frac{\mathrm{D}(\mathrm{D}+\mathrm{H} \sin \phi)^{2}}{\mathrm{~W}^{3}}
$$

or

$$
\frac{\partial A}{\partial D}=1-\frac{D+H \sin \phi}{W}+\frac{D H^{2} \cos ^{2} \phi}{W^{3}} \text {. }
$$

Now $W$ can be written in the form

$$
\mathrm{W}=\sqrt{(\overline{\mathrm{D}+\mathrm{H}} \sin \phi)^{2}+\mathrm{H}^{2} \cos ^{2} \phi .}
$$

Hence the sum to be subtracted from 1 in the expression for $\frac{\partial A}{\partial D}$ is greatest just when

$$
\mathrm{D}+\mathrm{H} \sin \phi=\mathrm{H} \cos \phi ;
$$

and in this case it is equal to

$$
\frac{1}{2}+\frac{1}{2}=1 \text {. }
$$

Thence it follows that $\frac{\partial A}{\partial \nu}$ is never $<0$. (We can also bring $\frac{\partial A}{\partial D}$ into the form

$$
1-\frac{(\mathrm{D}+\mathrm{H} \sin \phi)^{3}+2 \mathrm{H}^{2} \mathrm{D} \cos ^{2} \phi+\mathrm{H}^{3} \sin \phi \cos ^{2} \phi}{\mathrm{W}^{3}},
$$

from which we bring out the same conclusion by the development of W.)

But what has here been proved for $\frac{\partial A}{\partial \mathrm{D}}$ holds also for $\frac{\partial A}{\partial M}$, 
in case $D$ is very small in comparison with $M$; for the longitudinally magnetizing force is of the same nature as the direction-force. This is one reason why the work of the current for longitudinally magnetized is greater than for unmagnetic iron. But even when the force to be overcome is the same, the work is greater as soon as the angle between the direction of the force which does the work and the direction of the molecule at the commencement of the performance of work is greater. I will calculate at least the upper limit of this variation. Thus, let the molecule form with the axis the angle $\phi$; the first time let $\mathrm{H}$ act immediately and magnetize completely (that is, let the angle of rotation $\psi$ be $=\frac{\pi}{2}-\phi$ ); let this work be $A_{1}$. The next time let $M$ act first and magnetize completely, and consequently rotate through $\phi$; then let $\mathrm{H}$ act and on its part magnetize completely, rotating through $\frac{\pi}{2}$; let this work be $\frac{A_{2}}{A_{1}}$. The ratio $\frac{A_{2}}{A_{1}}$ can, evidently, never become greater than in this case, in which $M$ is of a higher order than $\mathrm{D}, \mathrm{H}$ of a higher order than $\mathrm{M}$. Now

$$
A_{1}=D(1-\sin \phi), \quad A_{2}=D ;
$$

therefore the mean values

that is,

$$
\begin{aligned}
& {\left[\mathrm{A}_{1}\right]=\frac{2 \mathrm{D}}{\pi} \int_{0}^{\frac{\pi}{2}}(1-\sin \phi) d \phi=\frac{\pi-2}{\pi} \mathrm{D},} \\
& {\left[\mathrm{A}_{2}\right]=. . . . . . . . . \mathrm{D} ;}
\end{aligned}
$$

$$
\frac{\left[\mathrm{A}_{2}\right]}{\left[\mathrm{A}_{1}\right]}=\frac{\pi}{\pi-2}=2 \cdot 752 \ldots
$$

Therefore, if $\mathrm{M}$ increases, $\mathrm{A}$ increases more rapidly than $M$ :- -first, because, with continually greater accuracy, $D$ can be neglected in comparison with $\mathrm{M}$; and hence the above formula for $\frac{\partial A}{\partial \bar{D}}$ becomes continually more strictly applicable; and, secondly, because the longitudinally magnetic state itself exerts a reaction, which, in rough approximation, can be taken into account by adding to $\mathrm{A}$ a factor constantly increasing in value with $M$ from 1 to $2.752 \ldots$ Whoever, up to these data, examines the observations that have been made on the extra currents, will find them qualitatively (and, as far as this 
is possible with the average insignificance of the deflections in general occurring, also quantitatively) verified.

I have characterized the extra currents in iron as the expression of the work of rotation, and described this work by an increase or a diminution of resistance. The value of the resistance corresponding to the closing current does not suddenly change into the value conditioned by the passage of a constant current or a permanent magnetization (or both together), but is mostly connected with it by a phenomenon of afteraction. Even of the nature of this, from the above an idea can easily be formed. If, beside the direction-force, only the principal current acts, it imparts to the molecules a rotation-velocity which, according to the value of $\mathrm{H}$, is constantly positive (rotation) or periodically changes its sign (oscillation). Now, if even in a magnetic needle moving in a copper shell we observe a rapid conversion of the motion of the mass into thermal motion, the same conversion will result much more rapidly still in a molecule of an iron wire. A heating therefore takes place, which is only gradually equalized by conduction and radiation; and the expression of this heating is the phenomenon of afteraction. In fact, heat is generated not merely by longitudinal, but (as Villari* has shown), also by circular magnetizing. Into this I do not further enter; I will only mention that the series of experiments $16-18$ of $\$ 4$ and the remarks (p. 16) in $\$ 5$ apply here. That in those experiments not only $\mathrm{D}$ and $\mathrm{H}$, but also $\mathrm{M}$ acted, has, it is selfevident, no essential influence upon the result. I add a few numbers characteristic of the course of this kind of afteraction.

(1) $\mathrm{M}=2 \mathrm{~B}, \mathrm{H}=1 \mathrm{D}, n=150$. These numbers were chosen so that $\delta$ was nearly $=0$. Wire $f_{4}^{\prime}$. The principal current was closed after the magnetizing current. $t$ denotes the time between two observations. On account of the considerable distance of the magnetizing current, some seconds mostly elapsed between the closing or opening of the magnetizing current and the first observation; hence the absolute alteration of resistance in consequence of the rotation-work cannot be inferred from the numbers. $s$ denotes closing, $\theta$ opening of the magnetizing current. The ratios are graphically represented in Pl. I. fig. 3 ; the reinforced parts of the drawing correspond to afteractions.

It will be seen that at the opening the duration of the afteraction is less than at the closing.

* Nuovo Cim. (2) iv. Nor.-Dec. 1870. 


\begin{tabular}{|c|c|c|c|c|}
\hline \multirow{2}{*}{$t$. } & & \multirow[t]{2}{*}{$w$} & \multicolumn{2}{|c|}{$\begin{array}{l}\text { Duration of the after- } \\
\text { action. }\end{array}$} \\
\hline & & & At closing. & At opening. \\
\hline \multirow{5}{*}{$\begin{array}{l}20^{\prime \prime} \\
20\end{array}$} & & 07906 & & \\
\hline & $\begin{array}{l}s \\
\ddot{o}\end{array}$ & $\begin{array}{l}0.7911 \\
0.7904\end{array}$ & $?$ & No oftaraction \\
\hline & $s$ & 0.7912 & 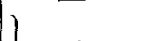 & 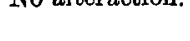 \\
\hline & & $0 \cdot 7907$ & $40^{\prime \prime}$ & \\
\hline & $\ddot{\sigma}$ & $\begin{array}{l}0.7905 \\
0.7903\end{array}$ & - & $80{ }^{-1}$ \\
\hline \multirow{5}{*}{$\begin{array}{l}20 \\
15 \\
15 \\
30\end{array}$} & & 0.7905 & - & $20^{\prime \prime}$ \\
\hline & $s$ & 0.7911 & 1 & \\
\hline & & 07909 & $30^{n}$ & \\
\hline & & $\begin{array}{l}07908 \\
07908\end{array}$ & - & \\
\hline & $\ddot{o}$ & 07905 & 一 & $35^{\prime \prime}$ \\
\hline \multirow{4}{*}{$\begin{array}{l}35 \\
\\
30 \\
20 \\
20\end{array}$} & & 07906 & 一 & $35^{\prime \prime}$ \\
\hline & $s$ & $\begin{array}{l}0.7911 \\
0.7906\end{array}$ & 1 & \\
\hline & & $0 \cdot 7905$ & $50^{\prime \prime}$ & \\
\hline & & 0.7905 & & \\
\hline \multirow{5}{*}{$\begin{array}{l}20 \\
40 \\
10 \\
20\end{array}$} & $\ddot{o}$ & $\begin{array}{l}0.7903 \\
0.7905\end{array}$ & $\bar{z}$ & $30^{\prime \prime}$ \\
\hline & $s$ & 0.7912 & 3 & \\
\hline & & $0 \cdot 7905$ & $50^{\prime \prime}$ & \\
\hline & & $\begin{array}{l}0.7904 \\
0.7904\end{array}$ & & \\
\hline & $\ddot{o}$ & 0.7901 & 一 & No afteraction. \\
\hline & & Mean ... & $45^{\prime \prime}$ & $15^{\prime \prime}$ \\
\hline
\end{tabular}

(2) Without magnetizing. $\mathrm{H}=2 \mathrm{D}$. A thick soft-steel wire, $\mathrm{F}_{6}$. The bridge in which was placed the galvanometer, the current being closed, and the current, the bridge being closed, were alternately closed and opened. In the first case there cannot in general be any extra current. (The weakening of the current in the wire by branching off into the galvanometer cannot, in my arrangement, have had any perceptible influence.) In the second case, on the contrary, the full current enters into the phenomenon. It was not powerful; nevertheless the afteraction was great. This was shown in the following way :- In the first case occurred a first excursion $s_{1}$ and a definitive deviation $u_{1}$; the two stood to one another in a constant ratio, conditioned only by the nature of the galvanometer-needle and the damping. In the second case an excursion $s_{2}$ occurred, greater than $s_{1}$, and, just as the needle had finished its periodic oscillations, a deviation $u_{2}$, likewise greater than $u_{1}$, which sank only gradually to $u_{1}$. In the Table, each row contains two experiments, viz. one of each sort.

$$
w+w_{k} \text { was }=0.2389 .
$$


the Galvanic Current through Iron.

\begin{tabular}{|c|c|c|c|c|c|}
\hline No. & $s_{1}$ & $u_{1}$. & $s_{2}$ & $u_{2^{2}}$ & $\begin{array}{l}\text { Siuks to } \\
\left(i i_{1}\right) .\end{array}$ \\
\hline $\begin{array}{l}1 \ldots \ldots \ldots \\
2 . \ldots \ldots \\
3 . \ldots \ldots \\
4 \ldots \ldots \\
5 \ldots \ldots\end{array}$ & $\begin{array}{l}4 \cdot 3 \\
4 \cdot 9 \\
5 \cdot 2 \\
5 \cdot 5 \\
5 \cdot 6\end{array}$ & $\begin{array}{l}2 \cdot 3 \\
2 \cdot 9 \\
3 \cdot 0 \\
3 \cdot 4 \\
3 \cdot 5\end{array}$ & $\begin{array}{l}5 \cdot 2 \\
6 \cdot 0 \\
6 \cdot 1 \\
6 \cdot 4 \\
6 \cdot 6\end{array}$ & $\begin{array}{l}4 \cdot 0 \\
3 \cdot 9 \\
3 \cdot 9 \\
4 \cdot 1 \\
4 \cdot 1\end{array}$ & $\begin{array}{c}2 \cdot 6 \\
2 \cdot 9 \\
? \\
3 \cdot 4 \\
?\end{array}$ \\
\hline Means... & $5 \cdot 1$ & $3 \cdot 0$ & $6 \cdot 1$ & $4 \cdot 0$ & $3 \cdot 0$ \\
\hline
\end{tabular}

Of the slow increase of all the numbers from experiment to experiment I shall speak presently.

Here belong also some facts already alladed to:- thus the phenomenon that, even in the cases in which the magnetizing diminishes the resistance, the first experiment gives the opposite result; for the evolution of heat occasioned by friction in the performance of work continues to operate. Further, the opening has often an influence in the opposite direction; that is, the resistance is lessened by magnetizing where otherwise it would be augmented, or is more strongly depressed than it would otherwise be. This phenomenon appears especially striking on the reversal of the current (compare $\$ 4$, experiment-series 10 and 11 ).

Some of the various phenomena just described may, I think, with more justice be designated as specific magnetic aftereffect*; but I here confine myself to a brief statement $\uparrow$. First, the intensity of the extra currents increases at the frequent passage of the current, or at frequent magnetization. This phenomenon has already been observed by Herwig; and he has explained it by an increasing mobility of the particles. The second of the above Tables shows it very clearly. At the same time it follows that the afteraction in consequence of the performance of work, which is the subject of that Table, does not simultaneously increase; for the numbers $u_{2}$ are nearly constant, and the difference $u_{2}-u_{1}$ sinks from $1 \cdot 7$, through $1 \cdot 0,0 \cdot 9,0 \cdot 7$, to $0 \cdot 6$. In connexion with this is the fact that the resistance of iron generally increases not inconsiderably on the current being repeatedly conducted through it. This phenomenon must not be confounded with that observed by Von Quintus Icilius-namely, that the resistance of all metals is increased after a single long-continued passage of a current through them. The phenomenon is much more pro-

* I see, from a memoir by Fromme, just published (Wied. Ann. iv. p. 76), that he also uses the same expression for analogous phenomena.

$\dagger$ Conf. Herwig, Streintz, l. c.; and further, Herwig, Pogg. Ann. clvi. p. 430 (1875).

Pliil. Mag. S. 5. Vol. 8. No. 47. Aug. 1879. 
nounced in iron the first time it is used galvanically, and mostly vanishes after the tenth to the hundredth closing. Thus the result with an iron wire $(l=1670, d=0 \cdot 20, \mathrm{H}=2 \mathrm{D}$, $M=1 D)$ was :-

$$
\begin{aligned}
& \text { Initially . . . . . } w=7 \cdot 3501 \\
& \text { After the 10th closing . . } 7 \cdot 3539 \\
& \text { "100th " . } 7 \cdot 3552 \text { (const.) } \\
& \text { Longitudinally magnetized . } \quad 7 \cdot 3547 \\
& \text { Magnetized ten times . . } 7 \cdot 3541 \\
& \text { Demagnetized . . . . } 7.3559
\end{aligned}
$$

These numbers show the afteraction with transverse magnetization by the current as well as with longitudinal magnetization. That the decrease of resistance in consequence of the latter appears so trifling (it became much greater at equal values of $\mathrm{H}, \mathrm{M}, n$ after the relations of the wire had become stationary), evidently depends on this-that the two afteractions, as indeed is mostly the case with these phenomena, do not go on independently of one another, and therefore, in consequence of the accelerated afteraction of the frequently repeated transverse magnetizing, a part of the decrease of resistance is concealed.

According to the preceding, it appears a probable supposition that the permanent alterations of resistance with the magnetic state, produced by magnetizing, may also be taken as the expression of certain performances of work. In fact, on a current passing through an iron wire, constant rotations of the molecules will be produced, which at an alteration of the arrangement, such as is produced by magnetizing, cannot remain unchanged. If these speculations are of a very hypothetical character, they nevertheless, I think, afford more fixed points than those of Beetz.

Attempts have been made by several to gain new fixed points by artificial transverse magnetization of the iron passed through by the current. I have not hitherto pursued this idea experimentally,-first, because we have not, as might be inferred from the hypotheses tacitly assumed by the authors in question, simply transverse opposed to longitudinal magnetization, but much rather here again, according to the arrangement, many different kinds of magnetization are conceivable (wherefore I have, for the sake of definiteness, designated the one here discussed as circular magnetization); and, next, because these experiments require still greater delicacy of measurement than experiments with longitudinal magnetization. The theory requires that artificial circular magnetizing (that is, additional to that produced by the principal current), 
if it alter the resistance at all, shall augment or diminish it according as the direction of the artificial is the same as or opposite to that of the natural circular magnetization, and according to their intensities. I am not aware of any experiment on this point. On the other hand, Sir W. Thomson* conducted the current through a square iron plate in a direction inelined to the direction of the magnetization. The poles of the electromagnet were situated at two opposite sides of tho square, the electrodes of the principal current in two opposite angles. Of the individual current-threads into which the plate under these circumstances divides, Thomson investigated the two meeting at the margin, each of which consists of two consecutive edges, A B C and A D C (fig. 4). The result of the experiment was, that the magnetization of the level-line D B was shifted into the position $D_{\epsilon}$, and consequently the resistance along $\mathrm{A} B$ was less than that along $\mathrm{A} D$, and the resistance along $\mathrm{DC}$ was less than that along $\mathrm{BC}$. Beetz and others have pointed out that even the mechanical pull connected with the magnetizing would by itself alone have necessitated this result. I think 1 must concur in this explanation: I will, however, show that my theory is not inconsistent with Thomson's observation, that, under some circumstances, it requires the latter, and that these circumstances were not present in the corresponding experiment made by Beetz $\dagger$ (which had a negative result).

Along the line A B, in Thomson's experiment, the directing force of the electromagnet will disturb the circular magnetization more or less, according to its intensity in comparison with that of the current-thread, and change it into a transverse magnetizing, in which all the molecular north poles point to the same side of the space. At the same time the resistance in certain lines of the current-thread, namely in those in which both the magnetizing forces have the same direction or make with one another an angle of $180^{\circ}$, will be but slightly changed; in the rest it will be generally diminished. Thus the total resistance will either be lessened or, if the electromagnet is powerful, somewhat but not much increased. But just in this latter case the resistance of the current-thread $\mathrm{BC}$ must very considerably increase; for here the electromagnet nullifies the circular magnetizing and produces a powerful longitudinal magnetization. From this, it is true, nothing can be inferred as to how the total resistance of $A B C$ varies; but just as little can be inferred from Thomson's experiments. It merely follows that the whole or the greatest portion of any decrease of the resistance, but only * Loc. cit. p. 741. 
the smallest portion of any increase, must fall upon the part $\mathrm{A} \mathrm{B}$; and this we in fact learn from the above consideration. That I have therein taken no account of the action of neighbouring current-threads makes no difference; for the result of this action is, for A B exactly as for B C, that the molecular magnets are brought into a position a little nearer the perpendicular to the plane of the plate.

In Beetz's experiment the iron-wire spiral to be magnetized was inside the magnetizing copper spiral, so that the turns of the one were parallel to those of the other. Consequently the molecules were more or less approximately so placed that (supposing the windings horizontal and the current flowing in the copper in the direction of motion of the hands of a watch) all the north poles pointed downward. The principal currerit, on the other hand, called forth a circular magnetizing; therefore in the portions of wire belonging to the front half of the iron spiral, although the principal current flowed in the direction in which the hands of a watch move, the north poles in the anterior semicylinders into wh $\mathrm{ch}$ each portion of the wire can be resolved were directed more or less upward, in the posterior (inner) downward. Conversely, in the portions of wire of the hinder half-spiral, the north poles in the posterior (outer) semicylinders were directed upward, and downward in the anterior ones. If, then, the magnetizing force of the principal current is not very little in comparison with the other, half of the current-threads present a stronger resistance than before the transverse magnetizing, the other half a weaker one; thus the total resistance remains nearly unaltered. The nogative result of Beetz's experiment is therefore not surprising.

[To be continued.]

XIX. On the Possibility of accounting for the Continuance of Recurring Changes in the Universe, consistently with the Tendency to Temperature-Equilibrium. By S. Tolver Preston*.

TTHE idea of the ultimate final cessation of all activity and 1 life in the universe has been contemplated by many physicists with some dissatisfaction, and with the desire, if possible, to find some explanation or physical means by which so apparently purposeless an end is averted, and of avoiding the necessity for assuming in past time a violation of physical principles at present recognized to exist. The allied notion of an unstable universe whose parts tend to agglomerate together into one mass by successively falling together, would certainly, to

* Communicated by the Author. 